ORDINES MILITARES

COLLOQUIA TORUNENSIA HISTORICA

Yearbook for the Study of the Military Orders

ISSN (print) 0867-2008 / ISSN (online) 2391-7512

DOI: http://dx.doi.org/10.12775/OM.2016.008

pp. $155-170$

UDO ARNOLD

Rheinische Friedrich-Wilhelm-Universität Bonn

Projektbereich Ostdeutsche Landesgeschichte

Am Hofgarten 22

D-53113 Bonn

Germany

UdoArnold@gmx.de

\title{
REFORMEN UND ERNEUERUNGEN IM DEUTSCHEN ORDEN VOM 16. BIS ZUM 20. JAHRHUNDERT
}

\author{
KeYwORDS \\ Military Orders; Teutonic Order; Order's Reform; Early Modern Time; Modern \\ Time
}

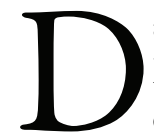

as Mittelalter verstand Reform als Rückführung auf Ursprüngliches. Im Deutschen Orden wird das gut sichtbar bei seiner Regelreform von I 442, die eine wörtliche Rückführung auf die Regel von I 249/ I 250 bedeutete. Das war noch möglich, weil die regionale Struktur des Ordens weitgehend der in der Mitte des I 3. Jahrhunderts entsprach; nur das Heilige Land war verloren. Das I6. Jahrhundert bot jedoch harte Einschnitte, die Erneuerungen zwingend notwendig machten. ${ }^{1}$

1 Zur allgemeinen Ordensgeschichte im Mittelalter zuletzt K. Militzer, Die Geschichte des Deutschen Ordens, Stuttgart 2012; zur Regelreform 1442 U. Arnold, Reformatorskie tendencje w zakonie krzyżackim w Prusach w I potowie XV wieku, Zapiski Historyczne 45 (1980), 4, S. 7-19; ders., Reformansätze im Deutschen Orden wäbrend des Spätmittelalters, in: Reformbemühungen und Observanzbestrebungen im spätmittelalterlichen Ordenswesen (Berliner historische Studien 14; Ordensstudien VI), hrsg. v. K. Elm, Berlin 1989, S. 139-152; Wiederabdruck in: U. Arnold, Deutscher Orden und Preußenland. Ausgewäblte Aufsätze anläßlich des 65. Geburtstages (Einzelschriften der Historischen Kommission für ost- und westpreußische Landesforschung 26), hrsg. v. B. Jähnig, G. Michels, Marburg 2005, S. 225-235.

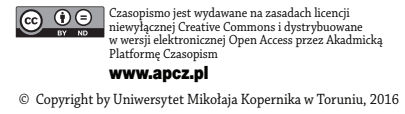




\section{i. Die Neuaufstellung des Ordens im Deutschen Reich im I6. JAHRHUNDERT}

I 525 verlor der Orden durch Säkularisierung und Lehnsnahme von der polnischen Krone seinen Hochmeister Albrecht von Brandenburg-Ansbach und mit ihm sein zentrales Territorium Preußen. Damit stellte sich die Frage, welcher Ordenszweig und welcher Meister in Zukunft die Führung übernehmen würde: Landmeister Wolter von Plettenberg mit einem durchaus beachtlichen Territorium in Livland oder Deutschmeister Dietrich von Cleen mit geringem Territorium und viel Streubesitz im Deutschen Reich. Zwar bedurfte die Frage einer aktuellen Entscheidung, doch war diese im Prinzip bereits vor I 525 zugunsten des Deutschmeisters gefallen. Die zunehmende Territorialisierung, die Tendenz zum frühmodernen Flächenstaat im Deutschen Reich bedingten einen deutlichen Druck der Zentralgewalt, die den Deutschmeister dazu brachte, sich in Schutz und Schirm des Reiches zu begeben, Leistungen für das Reich zu übernehmen und I 494 die Reichsfürstenwürde zu akzeptieren. Das widersprach zwar der Exemtion des I 3. Jahrhunderts, zeigt aber die Tendenz auf, der der Orden durch die erstarkenden Territorialfürsten unterworfen wurde.

Diese stärkere Eingliederung ins Reich machte den gedanklichen Weg frei für das Vorgehen nach dem Abfall des Hochmeisters. Noch ein Jahrhundert zuvor hatte der Orden auf dem Konstanzer Konzil mit Hilfe der Exemtion geschickt laviert zwischen Kaiser und Papst, nun setzte er eindeutig auf die Karte des Reiches, gegen die Kurie. Deutschmeister Dietrich von Cleen unterstanden acht Balleien, vier weitere - Österreich, Etsch, Elsass-Burgund und Koblenz - gehörten zum preußischen Hochmeistertum. Letztere waren plötzlich herrenlos und zeigten Selbständigkeitstendenzen, und innerhalb der deutschmeisterlichen Balleien gab es Zentrifugalkräfte besonders im Nordwesten, Biesen, Utrecht, Westfalen und Lothringen. Völlig klar war Cleen, dass drei eigenständige Ordensgebilde - also Deutschmeistertum, ein Block von vier abtrünnigen deutschmeisterlichen Balleien und ein Block aus vier hochmeisterlichen Balleien - nicht überleben, sondern der Territorialisierung durch die Landesfürsten zum Opfer fallen würden. Auch die Kurie in Rom konnte dabei nicht helfen. Es blieb nur die bewusste Fortsetzung der Einbindung ins Reich durch Anrufung der kaiserlichen Hilfe. Entgegen der römischen Aufforderung, einen neuen Hochmeister zu wählen, ${ }^{2}$ bemühte Cleen

2 Urkunde Papst Clemens VII. von 1526, Januar 31; Deutschordenszentralarchiv (DOZA) Wien, Urkunde zum Datum; Die Urkunden des Deutschordens-Zentralarchivs in Wien. Regesten, nach dem Manuskript von Marian Tumler hrsg. v. U. Arnold, Teil III (Quellen und 
sich - in abwehrender Konkurrenz zum livländischen Meister - um die kaiserliche Ernennung zum Administrator des Hochmeistertums in Preußen, als mit dem Deutschmeistertum verbundener Rechtsstellung. Als dies schließlich I 527 gelang, wurde die Urkunde als kaiserlicher Befehl deklariert, mit dem der Nachfolger Cleens, Walter von Cronberg, auch die vier hochmeisterlichen Balleien sich unterstellen konnte und so das Auseinanderbrechen des Ordens im Reich verhinderte. ${ }^{3}$ Damit war der Versuch des livländischen Landmeisters gescheitert, mit dem Argument des Kampfes gegen Moskau als Häretiker und Ketzer in Form eines Antemurale-Christianitatis-Denkens im Sinne Papst Leos X. die Ordensführung zu erlangen.

Der nächste Schritt war die Durchsetzung dieser neuen deutschmeisterlichen Stellung gegenüber allen Balleien auf einem Kapitel I 529, die Cronbergsche Konstitution. ${ }^{4}$ Diese Konstitution stellt einen Meilenstein im Wandlungsprozess des Ordens im Reformationszeitalter dar, der sich allerdings auf den Ordenszweig im Reich bezog. Livland blieb von dort aus gesehen ein Appendix, mit dem sich 1561/62 unter Landmeister Gotthard Kettler der parallele Vorgang wie in Preußen vollzog.

Studien zur Geschichte des Deutschen Ordens 60/III; Veröffentlichungen der Internationalen Historischen Kommission zur Erforschung des Deutschen Ordens 11/III), Marburg 2007, Nr. 4889.

3 Urkunde Kaiser Karls V. von 1527, Dezember 6; DOZA, Urkunde zum Datum; Die Urkunden (wie Anm. 2), Teil IV, Nr. 4929 (im Satz); Druck: Archieven der Ridderlijke Duitsche Orde, Balie van Utrecht, 2 Bde., hrsg. v. J. J. de Geer tot Oudegein, Utrecht 1871, Nr. 181; und F. de Salles, Annales de l'Ordre Teutonique ou de Sainte-Marie-de-Jerusalem depuis son origin jusquáa nos jours, Paris-Wien 1887 (Neudruck: Genève-Paris 1986), Nr. 161. Zu Cronberg vgl. A. Herrmann, Der Deutsche Orden unter Walter von Cronberg (1525-1543). Zur Politik und Struktur des „Teutschen Adels Spitale“ im Reformationszeitalter (Quellen und Studien zur Geschichte des Deutschen Ordens 35), Bonn-Bad Godesberg 1974; vgl. auch U. Arnold, Hochmeisterverlust, Bauernunruben und Reformation - Krisenbewältigung unter den Deutschmeistern Dietrich von Cleen und Walter von Cronberg, in: Die Ritterorden in Umbruchs- und Krisenzeiten. The Military Orders in Times of Change and Crisis (Ordines Militares. Colloquia Torunensia Historica XVI), hrsg. v. R. Czaja, J. Sarnowsky, Toruń 2011, S. 241-257.

4 Urkunde von 1529, September 1; DOZA, Urkunde zum Datum; Druck: de Geer (wie Anm. 3), Nr. 182; und Visitationen im Deutschen Orden im Mittelalter, Teil III (Quellen und Studien zur Geschichte des Deutschen Ordens 50/III; Veröffentlichungen der Internationalen Historischen Kommission zur Erforschung des Deutschen Ordens 10/III), hrsg. v. M. Biskup, I. Janosz-Biskupowa, u. der Redaktion v. U. Arnold, Marburg 2007, Nr. 249.

5 Vgl. U. Arnold, Hochmeister Albrecht von Brandenburg-Ansbach und Landmeister Gotthard Kettler - Ordensritter und Territorialherren am Scheideweg in Preußen und Livland, in: The Military Orders and the Reformation. Choices, State building, and the Weight of Tradition. Papers of the Utrecht Conference [der Internationalen Historischen Kommission zur Erforschung des Deutschen Ordens] 
Das deutschmeisterliche Vorgehen mit Blick auf das Reich blieb tragend für drei Jahrhunderte. Den Titel Administrator des Hochmeistertums in Preußen und Meister in deutschen und welschen Landen trug das Ordensoberhaupt bis I 835 , bis zur Neugliederung des Ordens nach Napoleon. ${ }^{6}$ Bei jedem Wechsel im Amt des Kaisers oder des Meisters erfolgte eine erneute Belehnung mit den Regalien durch den Kaiser, auch nach dem Zerbrechen des Römischen Reiches, zuletzt noch $1917 .^{7}$ Der in der Mitte des I 5 . Jahrhunderts einsetzende und 1494 in der ersten Verleihung der Regalien sichtbar gewordene Wandlungsprozess bot die Basis für die weitere Existenz des Ordens als nach außen wahrnehmbare Einheit. Dass dies Folgen für den Orden hatte, z.B. mit Leistungen für das Reich, war natürlich. Doch in einem Punkt trafen sich plötzlich die mittelalterliche Heidenkampfideologie des Ordens und die geforderten Leistungen für das Reich, im Abwehrkampf gegen die über den Balkan bis vor Wien heranrückenden Türken. Das führte letztlich zur Aufstellung eines eigenen Deutschordensregiments am Ende des I7. Jahrhunderts, von dem Traditionsstränge bis in die österreichische Gegenwart laufen. ${ }^{8}$

\section{Die KONFESSIONELle ReForm DeS i6. BIS i 8. JAHRHUNDERTS - TRIKONFESSIONALITÄT}

Es wäre geradezu verwunderlich gewesen, wäre die Konfessionsfrage am Orden vorbeigegangen. Die gelehrten theologischen Auseinandersetzungen bewogen sicher die wenigsten Ordensbrüder zum Konfessionswechsel. Aber gerade die Priester verließen den Orden, konnten sie doch von auf Armut verpflichteten Ordensbrüdern nun als Protestanten eigenständige Pfarrherren werden, die ihre Pfründen selber genossen. Der Ritter besaß als Komtur bereits eine Pfründe, der

(Bijdragen tot de Geschiedenis van de Ridderlijke Duitsche Orde, Balije van Utrecht 3), hrsg. v. J. A. Mol, K. Militzer, H. Nicholson, Hilversum 2006, S. 11-29.

6 Vgl. U. Arnold, Deutschmeister, Administrator des Hochmeistertums in Preußen, Hoch-und Deutschmeister, in: Herrschaft, Netzwerk, Brüder des Deutschen Ordens in Mittelalter und Neuzeit. Vorträge der Tagung der Internationalen Historischen Kommission zur Erforschung des Deutschen Ordens in Marburg 2010 (Quellen und Studien zur Geschichte des Deutschen Ordens 72; Veröffentlichungen der Internationalen Historischen Kommission zur Erforschung des Deutschen Ordens 12), hrsg. v. K. Militzer, Weimar 2012, S. 159-175, hier S. 170 f.

7 Urkunde von 1917, Mai 10; DOZA, Urkunde zum Datum.

8 Vgl. B. Demel, Der Deutsche Orden und das Regiment Hoch-und Deutschmeister von 1696 bis 1998. Überblick und neue Erkenntnisse, in: ders., Der Deutsche Orden einst und jetzt. Aufsätze zu seiner mehr als 800jährigen Geschichte (Europäische Hochschulschriften III/848), hrsg. v. F. Vogel, Frankfurt/Main u. a. 1999, S. 85-115. 
Orden sorgte für ein standesgemäßes Einkommen. Damit leistete der Deutsche Orden - genau wie Johanniter und Domkapitel - einen wichtigen Beitrag für die Versorgung der nachgeborenen Söhne vor allem des niederen Adels, als Spital des deutschen Adels.

Das soziale Problem des Adels blieb auch beim Wechsel der Konfession dasselbe. Deshalb setzten zwar die protestantischen Landesherrn den Wechsel der Konfession für Ritterorden und Domkapitel in ihrem Territorium durch, enteigneten sie jedoch nicht. Das bedingte ein erhebliches Umdenken innerhalb des Ordens. Wollte er die Balleien Hessen, Thüringen, Sachsen und Utrecht nicht verlieren, musste er den vom Landesherrn erzwungenen Konfessionswechsel akzeptieren. Das gestaltete sich für das lutherische Bekenntnis einfacher - lag es doch trotz allen Streites dem Katholizismus nicht so fern - als für den reichsrechtlich nach dem Westfälischen Frieden I 648 offiziell zugelassenen Calvinismus. Doch selbst das gelang in der jeweils zur Hälfte mit Lutheranern und Calvinisten sowie zusätzlich einem Katholiken besetzten Ballei Hessen. ${ }^{9}$

Das geradezu revolutionäre Denken dieser Reform zeigt sich am besten am Beispiel der Ballei Utrecht. In der Mitte des I6. Jahrhunderts hatte sich in den nördlichen Teilen der zu Spanien gehörenden Niederlande der Calvinismus weitgehend durchgesetzt, wenngleich die Landkomture weiterhin katholisch blieben. Die politische Entwicklung während des seit I 568 andauernden 80jährigen Krieges der nördlichen Niederlande gegen Spanien erzwang jedoch von der Ballei den Konfessionswechsel, I6 99 wurde mit Jasper van Linden der erste Nichtkatholik Landkomtur. ${ }^{10}$ Zwei Jahrzehnte später löste sich die Ballei endgültig aus dem Gesamtorden, wenngleich Versuche zur Wiedervereinigung bis zum Beginn des 19. Jahrhunderts von beiden Seiten unternommen wurden. ${ }^{11}$ Selbst mit der Tatsache, dass die Utrechter Ritter verheiratet waren, vermochte die Ordensleitung umzugehen. Der jesuitisch erzogene Deutschmeister Johann Caspar von

9 Vgl. ders., Von der katholischen zur trikonfessionellen Ordensprovinz. Entwicklungslinien in der Personalstruktur der hessischen Deutschordensballei in den Jahren 1526-1680/81, in: Elisabeth, der Deutsche Orden und ibre Kirche. Festschrift zur 700jährigen Wiederkehr der Weihe der Elisabethkirche Marburg 1983 (Quellen und Studien zur Geschichte des Deutschen Ordens 19), hrsg. v. U. Arnold, H. Liebing, Marburg 1983, S. 186-281; ders., Die bi- und schließlich trikonfessionelle Ballei Hessen des Deutschen Ordens zwischen Luther und Napoleon und die Ordenspfarre Reichenbach in Nordhessen von 1207 bis 1809, in: ders., 1190-2010. 820 Jahre Deutscher Orden. Fünf Referate über eine lange Geschichte in Europa, Wien 2011, S. 26-79.

10 Vgl. D. Grögor-Schiemann, Die Deutschordensballei Utrecht während der Reformationszeit: Die Landkommende zwischen Rebellion und Staatsbildung (Quellen und Studien zur Geschichte des Deutschen Ordens 68), Weimar 2015.

11 R. E. de Bruin, Bedreigd door Napoleon. De Ridderlijke Duitsche Orde, Balije van Utrecht 1753-1838 , Hilversum 2012. 
Ampringen sprach hinsichtlich des im Orden nach wie vor geltenden Gelübdes der Keuschheit für die Utrechter Ritter von Castitatem in Connubio, wozu Eheleute sowieso aus göttlichem Befehl verpflichtet seien. ${ }^{12}$ Martin Luther hatte dies in seinem Sendschreiben an den Orden von 1523 die rechte eheliche Keuschheit genannt. ${ }^{13}$ In dieser Konstruktion für Utrecht zeigt sich der Wandel des Ordens in der konfessionellen Frage wohl am deutlichsten, gleichzeitig die Nähe zum trikonfessionellen Reich und die Ferne zu Rom.

\section{Der geistige Neubeginn des i 7. Jahrhunderts}

Das Fundament des Ordenslebens bildete die Regel. Sie war 350 Jahre nach ihrer ersten Formulierung sicherlich nicht mehr zeitgerecht. In vielen Punkten hatte der Orden sich seit seiner Gründung gewandelt, das geistliche Fundament galt es neu zu durchdenken. Das Ergebnis war I 606 eine straffere, kürzere Fassung von Regel und Armutsgelübde: Privateigentum war de facto gegeben durch den vollen Genuss der Einkünfte des jeweiligen Amtes. Das führte in Konsequenz zur Testierfähigkeit, die jeder Ritter gegen eine gewisse Zahlung an den Orden erhielt, womit er privates Vermögen erwerben und vererben konnte. Das standesgemäße Leben eines Adeligen war damit gesichert, der seit dem I 5 . Jahrhundert erkennbare Wandel des Ordens zum Hospital des deutschen Adels auch durch das neue Ordensbuch abgesichert. Es handelte sich um eine adlige Ritterregel, die Priester spielten eher die Rolle eines Annexes. Die mittelalterliche Verfürstlichung des Hochmeistertums fand hier ihre Fortsetzung für jeden einzelnen Ritter. Allerdings wurde dem persönlichen Gebet, unmittelbar nach der Grundlegung der drei Gelübde, ein erheblicher Stellenwert eingeräumt, auch wenn es sich dabei nicht um die persönliche Hinwendung zu Gott im Sinne Martin Luthers handelte. ${ }^{14}$

Die zahlenmäßige Reduzierung der Ordensmitglieder im i 5. und i6. Jahrhundert hatte auch für die Priester eine Bildung von Konventen schon lange nicht

12 Druck der entsprechenden Verhandlungsinstruktion bei B. Demel, Rekuperationsbemühungen des Deutschen Ordens um die ihm entzogene Ballei Utrecht in den Jahren 1667-1685, in: Flores considerationum amicorum. Festschrift für Carl August Lückerath zum 70. Geburtstag am 13. Dezember 2006, hg. v. W. Hasberg, J. Schröder, Zürich 2006, S. 81-103, Anhang I.

13 M. Luther, An die herrn Deutschs Ordens das sie falsche keuscheyt meyden und zur rechten ehlichen keuscheyt greyffen Ermanung, Wittenberg 1523; moderner Druck: D. Martin Luthers Werke. Kritische Gesamtausgabe, Bd. 12, Weimar 1891, S. 228-244.

14 Vgl. Sammlung der neuesten Regeln, Statuten und Verwaltungsvorschriften des deutschen Ritterordens. 1606 bis 1839, Wien 1840, S. 73-88, bes. S. 77 f.; H. Noflatscher, Glaube, Reich und Dynastie. Maximilian der Deutschmeister 1558-1618 (Quellen und Studien zur Geschichte des Deutschen Ordens 11), Marburg 1987. 
mehr erlaubt. Hier wenigstens versuchte Hochmeister Erzherzog Maximilian nachzubessern. Ordenspriester wurde man erst nach der Priesterweihe, das mittelalterliche System von Schülern und Novizen vor der Priesterweihe war entfallen und ließ sich 1606 auch nicht wieder erneuern. Stattdessen wurde am Sitz des Meisters in Mergentheim ein Priesterseminar gegründet. Ähnliche Bestrebungen lassen sich vor allem in der Ballei Biesen mit Köln und Löwen feststellen, im Ansatz auch für die Ballei Elsass in Altshausen. ${ }^{15}$ Doch das System der dem Orden inkorporierten Pfarren ermöglichte keine Konventbildung der Priester. Dementsprechend war die Bindung an den Gesamtorden meist recht gering.

\section{Der Neubeginn im habsburgischen Reich des i9. Jahrhunderts}

Das Heilige Römische Reich deutscher Nation zerbrach unter dem Zugriff Napoleons und mit der Stärkung der einzelnen territorialfürstlichen Interessen. ${ }^{16}$ Für die Ritterorden erfolgte Enteignung und Vertreibung. Diese erneute Säkularisation bedeutete das Verschwinden auch des Deutschen Ordens von der Landkarte der deutschen Fürstentümer. Da der Hochmeister ein habsburgischer Prinz war, blieb zumindest in der österreichischen Monarchie seine Existenz für das nächste Vierteljahrhundert in der Schwebe, wenngleich das Aussterben des Ordens drohte. Erst I 834 schufen kaiserliche Bestimmungen, vorbereitet durch Staatskanzler Metternich, die Grundlage für die weitere Existenz des Ordens, gekennzeichnet durch die Aufnahme neuer Ritter. Dem folgte die Erarbeitung neuer Regeln und Statuten. Dabei wurde die Regel von 1606 in die neue Fassung mitaufgenommen. Gerade hinsichtlich des Armutsgelübdes wird eine weitere Entleerung erkennbar, verblieb doch nunmehr der Ritter voll im Genuss seines Vermögens - ein Faktum, das bis zum Tode des letzten Ritters I 970 unter Priestern noch Ärger erregte. Diese Regel von I 839 war wie ihre Vorläuferin eine adlige Ritterregel. ${ }^{17}$

15 Vgl. B. Demel, Das Priesterseminar des Deutschen Ordens zu Mergentheim (Quellen und Studien zur Geschichte des Deutschen Ordens 12), Bonn-Bad Godesberg 1972; ders., Zur Priestergewinnung und Seminarausbildung im Deutschen Reich und im schlesisch-mährischen Meistertum zwischen Reformation und Säkularisation, in: Priester im Deutschen Orden.Vorträge der Tagung der Internationalen Historischen Kommission zur Erforschung des Deutschen Ordens in Wien 2012 (Quellen und Studien zur Geschichte des Deutschen Ordens 77; Veröffentlichungen der Internationalen Historischen Kommission zur Erforschung des Deutschen Ordens 15), hrsg. v. U. Arnold, Weimar 2016, S. 157-175.

16 Vgl. F. Täubl, Der Deutsche Orden im Zeitalter Napoleons (Quellen und Studien zur Geschichte des Deutschen Ordens 4), Bonn 1966.

17 Sammlung (wie Anm. 14). 
Doch worauf konnte der Orden sich jetzt noch stützen? Grundbesitz zum Lebensunterhalt der in Diensten der Monarchie stehenden Ritter, Pfarren als Aufgabe für die verbliebenen Priester - das konnte nicht genug sein. Das Zeitalter des Übergangs von der Agrar- zur urbanisierten Industriegesellschaft, vom Heimgewerbe zur Hochindustrialisierung schuf erhebliche Probleme: soziale Umbrüche der Gesellschaft bis hin zum Pauperismus, dadurch bedingt auch politische Umbrüche bis zur Revolution, die Entwicklung von Liberalismus und Sozialismus, um nur wenige Bereiche anzusprechen. Der Fürstenherrschaft, den Orden und der bürgerlichen Gesellschaft fiel die Aufgabe zu, diese Probleme zu meistern. Die katholische Kirche bezog erst I 89 I mit der Enzyklika Rerum novarum durch Papst Leo XIII. Position dazu. Das Aufgreifen etlicher sozialer Probleme durch die Hochmeister aus habsburgischem Hause bot die neue Fundierung für den Orden, aber auch einen auf die mittelalterliche Tradition gestützten Wandel letztlich zu einer Entadeligung des Ordens, die im 20. Jahrhundert ihren Abschluss finden sollte.

Der neue Hochmeister Maximilian Joseph von Österreich-Este dachte an ein Aufgeben des militärischen Elements des Ordens, er definierte Rittertum neu. ${ }^{18}$ Dieser Bruch mit einer seit I 98 tragenden Tradition stellt einen enormen mentalen Wandel dar, der sich jedoch gegen die Ritter nicht durchsetzen ließ. So widmete der Hochmeister sich seit I 837 in einem ersten Schritt der Wiedererrichtung des mittelalterlichen Schwesternzweiges, was schließlich i 854/1855 abgeschlossen werden konnte. ${ }^{19}$ Damit hatte er eine Aufgabe, die sich im Rittertum nicht mehr verwirklichen ließ, auf eine neue Personengruppe übertragen. Die Aufgaben im sozialen wie im schulischen Raum haben den Schwestern viele Wirkungsfelder bis in die Gegenwart geboten. Gleichzeitig hielt das weibliche Element erneut Einzug im Orden. Dies prägte nicht nur sein Erscheinungsbild nach außen, sondern entwickelte auch einen quasi geschwisterlichen Umgang von Brüdern und Schwestern bis in die Gegenwart.

Der nächste Schritt galt der Versorgung der inkorporierten Pfarren mit eigenen Ordenspriestern, wozu der Hochmeister als Helfer den Trienter Theologieprofessor Peter Rigler heranzog. So konnte 1855 in Lana in Tirol der erste Priesterkonvent begründet, damit ein Ansatz der Priesterausbildung von 1606

18 Vgl. Erzherzog Maximilian Joseph von Österreich-Este. Hochmeister - Festungsplaner - Sozialreformer - Bildungsförderer (1782-1863). Mit Beiträgen zum Deutschen Orden in Linz und Oberösterreich (Quellen und Studien zur Geschichte des Deutschen Ordens 69), hrsg. v. E. Volgger, Linz-Weimar 2014.

19 Vgl. E. Gruber, Deutschordensschwestern im 19. und 20. Jahrhundert. Wiederbelebung, Ausbreitung und Tätigkeit 1837-1971 (Quellen und Studien zur Geschichte des Deutschen Ordens 14), Bonn-Bad Godesberg 1971. 
wieder aufgegriffen werden. Ihm folgten Konvente in Österreichisch-Schlesien. Hier entstand der Ordenspriester neuen Stils, der schließlich i 87 I seine eigene Regel erhielt. ${ }^{20}$

Der dritte Schritt sollte das Heranziehen von Laien für die Ordensarbeit sein, denen gegen geistige oder materielle Hilfe das Ordenskreuz verliehen werden konnte. Die Realisierung dieser Vorstellungen erfolgte allerdings erst unter Maximilian Josephs Nachfolger Erzherzog Wilhelm mit der Gründung der Institute der Ehrenritter I 865 und der Marianer I871. Gedacht waren sie zur Unterstützung des neu errichteten Hospitaldienstes in Krieg und Frieden, der im Serbisch-Bulgarischen Krieg von i 885/ 1886 seinen ersten Einsatz und im Ersten Weltkrieg einen Höhepunkt fand. ${ }^{21}$ Hier liegt der entscheidende Wandel darin, dass aufgrund der notwendigen Spezialisierung im Hospitaldienst der Orden nicht mehr mit eigenen Mitgliedern die Aufgaben erfüllen konnte, sondern weltliche, zu entlohnende Mitarbeiter benötigte, die unter seinem Namen tätig werden. Die modernen Deutschordenswerke arbeiten auf dieser Grundlage.

\section{Zusammenbruch Und NeUbeginn IM 20. Jahrhundert}

Enteignung und Vertreibung bei der Auflösung des Heiligen Römischen Reichs Deutscher Nation schienen bereits einen negativen Höhepunkt der Ordensentwicklung dargestellt zu haben, doch das 20. Jahrhundert hatte noch Schlimmeres zu bieten. Die Auflösung des Habsburgerreiches als Ergebnis des Ersten Weltkrieges beraubte den Orden seiner bisher wichtigsten Stütze. Das Ringen um die Form zukünftiger Ordensexistenz ist sicher eins der spannendsten, wenngleich nach wie vor nicht genügend aufgearbeiteten Kapitel. ${ }^{22}$ Der mit der Kurie abgesprochene Verzicht des Hochmeisters Erzherzog Eugen auf sein Amt bedeutete den Übergang der Ordensleitung auf einen Priester. Das stellt den Bruch einer seit I 198, seit der Umwandlung des Hospitals der Deutschen in Akkon in eine ritterliche Korporation kontinuierlich fortgesetzten Form dar. Der Ritterorden gab sein jahrhundertealtes Profil auf, er wandelte sich zu einem priesterlich-karitativen Institut. Vorbereitet war dies im 19. Jahrhundert durch die Einführung des Schwestern-

20 Vgl. U. Gasser, Die Priesterkonvente des Deutschen Ordens. Peter Rigler und ihre Wiedererrichtung 1854-1897 (Quellen und Studien zur Geschichte des Deutschen Ordens 28), Bonn-Bad Godesberg 1973.

21 Vgl. G. Müller, Die Familiaren des Deutschen Ordens (Quellen und Studien zur Geschichte des Deutschen Ordens 13), Marburg 22010.

22 Vgl. A. O. Wieland, Wandel im 20. Jahrhundert: Vom Ritterorden zum klerikalen Orden, in: Priester im Deutschen Orden (wie Anm. 15), S. 177-214. 
instituts und die Reform der Priesterausbildung in Konventen. Dies bedeutete zwar eine stärkere Eigenständigkeit der Brüderprovinzen, doch das Wirken der Schwestern erhielt damit im Erscheinungsbild des Ordens einen noch höheren Stellenwert als bisher.

Der Zerfall des Habsburgerreiches zog eine Nationalisierung der Nachfolgestaaten nach sich, innerhalb des Ordens am stärksten im slowenischen Raum zu spüren, aber auch in der Tschechoslowakei. Der Orden wurde immer mehr als Träger des Deutschtums in einem anders ausgerichteten Staat angesehen. Hinzu kamen Anklagen in Rom, die 1928, mitten im Prozess der mentalen Neuaufstellung des Ordens, zur Ernennung eines mit allen Vollmachten bis hin zur Auflösung ausgestatteten Visitators führten.

Als wichtigste Persönlichkeit entwickelte sich P. Marian Tumler, der als promovierter Historiker, seit 1923 Ordensarchivar, die Spezifika des Ordens zu retten versuchte. ${ }^{23}$ Dass das Rittertum nicht mehr zu halten war, bedauerte er zwar, akzeptierte es jedoch. Auf jeden Fall wollte er die Schwestern - entgegen den römischen Vorstellungen - als Teil des Ordens erhalten, stellten sie doch zahlenmäßig wie in der Vielfalt ihrer Aufgabenbereiche den bedeutendsten Anteil der Ordenstätigkeit. Gleichzeitig ging es ihm um den Erhalt der dem Orden nicht mit Profess verbundenen Helfer, auch das ein umstrittenes Vorhaben. Beides konnte schließlich in der neuen Regel 1929 verankert werden, so dass die innere Struktur des Ordens mit einem Brüderzweig, einem Schwesternzweig und Familiaren ohne Profess viele Kontinuitäten aus seiner Geschichte aufwies.

Zwar stellten sich die Anklagen in Rom als unbegründet heraus, doch der Orden benötigte zu jedem einzelnen Schritt die Genehmigung des Visitators, der erst 1938 seines Auftrags entbunden wurde, drei Wochen nach dem sogenannten "Anschluss" Österreichs an das nationalsozialistische Deutsche Reich.

Damit aber trat die bislang sehr gehemmte Neuaufstellung des Ordens in eine noch kritischere Phase. Die Kirchenfeindlichkeit des Nationalsozialismus verband sich mit der im 19. Jahrhundert im Königreich Preußen und im deutschen Kaiserreich erfolgten Traditionsaneignung der Deutschordensgeschichte und den daraus erwachsenden Plänen eines eigenen „Deutschen Ordens“. ${ }^{24}$ Dem stand der

23 Vgl. U. Arnold, Dr. Marian Tumler, in: Die Hochmeister des Deutschen Ordens 1190-2012 (Quellen und Studien zur Geschichte des Deutschen Ordens 40), hrsg. v. dems., Weimar ${ }^{2} 2014$, S. 330-336; russ. Ausgabe: Moskau 2015, S. 327-333; ders., Hochmeister P. Dr. Marian Tumler (1887-1987), in: Priester im Deutschen Orden (wie Anm. 15), S. 215-230.

24 Vgl. U. Arnold, Nationalismus, Nationalsozialismus und der Mißbrauch der Deutschordenstradition in Deutschland, in: Der Deutsche Orden und die Ballei Elsaß-Burgund. Die Freiburger Vorträge zur 800-Jahr-Feier des Deutschen Ordens, hrsg. v. H. Brommer, Bühl/Baden 1996, S. 205-222; ders., Der Deutsche Orden im deutschen Bewußtsein des 20. Jahrhunderts, in: Vergangen- 
real existierende und durch den Anschluss Österreichs 1938 und großer Teile der Tschechoslowakei 1939 quasi „geerbte“ Deutsche Orden im Wege. Konsequent waren das Verbot und die Enteignung in Österreich und im Sudetenland; die anschließende kommunistische Herrschaft in der Tschechoslowakei übernahm sowohl Geschichtsverdrehung als auch Enteignung durch die Nationalsozialisten bis heute. Das staatliche Vorgehen bedeutete einen Exodus von Priestern und Schwestern aus der Tschechoslowakei sowie Verfolgung in Jugoslawien - der Orden unterlag einem Martyrium staatlicherseits. ${ }^{25}$

Flucht und Vertreibung aus der Tschechoslowakei führten ab I $945 \mathrm{zu}$ neuen Niederlassungen von Schwestern und Brüdern vor allem in der allmählich entstehenden Bundesrepublik Deutschland, aber auch in Österreich, wo der Orden I 947 seine Restituierung erreichen konnte. Der keineswegs problemlose Neuaufbau traf auf die staatlich-gesellschaftliche Entwicklung der soer und 6oer Jahre. Sie bedeutete in kirchlicher Hinsicht eine Säkularisierung und Individualisierung, die auch die Orden immer stärker betraf. Das Zweite Vatikanische Konzil hat vor 50 Jahren vorsichtig versucht, dem Rechnung zu tragen, wenn Papst Johannes XXIII. in seiner Eröffnungsansprache betonte, dass es sehr auf die Würde der menschlichen Person und die daraus folgenden Verpflichtungen ankommt. ${ }^{26}$

Der Deutsche Orden konnte die mit der sozialen Entwicklung verbundenen Probleme aufgrund seiner traditionsbezogenen Führung unter Hochmeister Tumler und seiner besonderen, dreigliedrigen Struktur lange Zeit überdecken. Tumler gelang es sogar, 1965 seitens der Kurie die Anerkennung der Familiaren als eigenes, dem Orden affiliiertes Institut zu erhalten. Waren die mittelalterlichen Familiaren wie auch die Ehrenritter und Marianer des i 9. Jahrhunderts nur auf das Recht des Ordens gegründet und eine Hilfsinstitution von Einzelpersonen, so wurden die modernen Familiaren nun noch enger an den Orden gebunden. Sie bilden keine eigenständige Institution wie etwa der 3. Orden des hl. Franziskus, sondern stehen ebenfalls unter der Leitung des Hochmeisters mit einer eigenen Gemeinschaftsstruktur. Das päpstliche Familiarenstatut von 1965 ist voll in Übereinstimmung

heit und Gegenwart der Ritterorden. Die Rezeption der Idee und die Wirklichkeit (Ordines Militares. Colloquia Torunensia Historica XI), hrsg. v. Z. H. Nowak u. Mitarbeit v. R. Czaja, Toruń 2001, S. 39-53; Wiederabdruck in: ders., Deutscher Orden und Preußenland (wie Anm. 1), S. 93-106. 25 Für die Schwestern vgl. E. Gruber, Hinter dem Eisernen Vorhang. Deutschordensschwestern in der ČSR/ČSSR 1945 bis 1989 (Quellen und Studien zur Geschichte des Deutschen Ordens 71), Weimar 2012.

${ }^{26}$ Zum Folgenden vgl. Peregrinantes Peregrinantibis. 825 Jahre Deutscher Orden, 150 Jahre Ehrenritter, 50 Jahre Familiarenstatut (Quellen und Studien zur Geschichte des Deutschen Ordens 80), hrsg. v. U. Arnold, B. Huber, Weimar 2018, u. a. den Beitrag von D. Salch, 150 Jahre Ehrenritter, 50 Jahre Apostolisches Familiareninstitut; und von U. Arnold, Hochmeister Marian Tumler und das Zweite Vaticanum. 
mit den Tendenzen des II. Vatikanischen Konzils zu verstehen zur stärkeren Beteiligung des Laien in der Kirche. Das Konzil verabschiedete gerade in jenem Jahr ein entsprechendes Konzilsdokument Apostolicam actuositatem. Damit besitzt der Orden nunmehr drei Zweige, wenngleich abgestuften Rechts: Brüder, Schwestern und Familiaren.

Gleichzeitig erhielt der Orden aber auch einen neuen Auftrag durch das Konzilsdokument Perfectae caritatis, das Dekret über die zeitgemäße Erneuerung des Ordenslebens. Damit bedurfte es wiederum einer neuen Ordensregel, für Hochmeister Tumler die zweite, an der er wesentlichen Anteil hatte. Sie behielt die 1929 gewonnene Struktur des Ordens unter stärkerer Einbindung der Familiaren bei, doch glücklich war Tumler mit dem Ergebnis keineswegs: Den Bearbeitern der Entwürfe mußte schon bei Beginn ihrer Arbeit das Recht eingeräumt werden, nach dem Muster und den Weisungen des Konzils zu arbeiten. Daß dabei etwas allen Alten im Orden völlig Fremdes herauskommen mußte, etwas ganz anders Geartetes als unsere "alten "Regeln waren, ist selbstverständlich. Wir Alten und auch das Generalkapitel haben sich damit abgefunden. Diese neuen, 1977 von Rom approbierten Lebensregeln der Brüder sind kürzer, enthalten weniger Detailregelungen und fordern aufgrund einer Weitmaschigkeit der Vorschriften mehr an persönlicher Entscheidung und Verantwortung. Die Lebensregeln der Schwestern dagegen sind ausführlicher als I 929 und stehen in vielem gleichberechtigt neben den Brüderregeln, auch wenn der Anspruch auf die Ordensleitung durch die Brüder voll akzeptiert wird. Insgesamt kann man der Feststellung einer Schwester folgen: Was unsere neue Regel besonders charakterisiert, ist das demokratische Denken. ${ }^{27}$ Diese Demokratisierung ist sicher ein wesentliches Erneuerungsmoment innerhalb des Ordens, auch wenn dadurch das Gelübde des Gehorsams nicht entwertet wird. Völlig neu ist dieser Ansatz jedoch auch nicht, wenn an die bereits in der Regel aus der Mitte des I 3 . Jahrhunderts festgeschriebene Ratspflicht aller Oberen erinnert werden darf. ${ }^{28}$ Daneben stehen mit eigenen Lebensvorschriften, wenngleich ohne Profess, die Familiaren.

\section{Schluss}

Damit sind die Reformen und Erneuerungen des Deutschen Ordens in ihren großen Schritten bis in die Gegenwart dargestellt. Sie waren weitgehend durch die

27 B. Platter, Werdegang und Charakterisierung der nachknziliaren Schwesternregeln, in: Die Regeln des Deutschen Ordens in Geschichte und Gegewart, hrsg. v. E. Volgger, Lana 1985, S. 303-327, hier S. 324

28 Die Statuten des Deutschen Ordens, hrsg. v. M. Perlbach, Halle/Saale 1890, Regel, Kapitel 27, S. 49. 
politischen Ereignisse aufgezwungen. Erst die letzte Reform erfolgte aufgrund eines kirchlichen Anstoßes, wenngleich die Ursache dafür ein deutlicher gesellschaftlicher und innerkirchlicher Wandel war. Das macht klar, dass Reform und Erneuerung innerhalb eines Ordens, und damit auch des Deutschen Ordens, einen fortlaufenden Prozess darstellen. Es ist eine Entwicklung in vielen kleinen Schritten, die keineswegs so spektakulär sind wie die hier geschilderten, aber aufgrund der politischen und gesellschaftlich-sozialen Umstände ständige Reaktionen fordern. So wird beispielsweise seit der letzten Regelrevision ein deutlicher Wandel erkennbar. Den zahlenmäßig stärksten Zweig des Ordens stellen heute nicht mehr die Schwestern, sondern die Familiaren. Auch durch ihre Initiativen wird die Tätigkeit des Ordens immer mehr von weltlichen Mitarbeitern getragen, nicht mehr von Ordensmitgliedern - ein Wandel, der bereits im Hospitalwesen des I 9. Jahrhunderts angebahnt wurde. Die politisch-soziale Entwicklung bis in unsere Gegenwart überrascht immer wieder mit neuen Problemen. Die Wandlungen um den Orden herum, die ebenso Auswirkungen auf sein Innenleben haben, laufen in einem enormen Tempo ab und fordern stets neue Antworten. Daher wird das hier Geschilderte in einigen Jahren mit Sicherheit ein zusätzliches, neues Kapitel erfordern.

\section{QUELLEN- UND LITERATURVERZEICHNISS}

"D. Martin Luthers Werke. Kritische Gesamtausgabe." Vol. I 2. Weimar: Böhlau, I 89 I.

Luther, Martin. An die herrn Deutschs Ordens das sie falsche keuscheyt meyden und zur rechten eblichen keuscheyt greyffen Ermanung. Wittenberg: Rhau-Grunenberg, I 523.

Perlbach, Max., edit. "Die Statuten des Deutschen Ordens." Halle/Salle: M. Niemeyer, I 890. "Sammlung der neuesten Regeln, Statuten und Verwaltungsvorschriften des deutschen Ritterordens. 1606 bis I 839 ." Wien: Sollinger, 1840.

Arnold, Udo. "Der Deutsche Orden im deutschen Bewußtsein des 20. Jahrhunderts." In Vergangenheit und Gegenwart der Ritterorden. Die Rezeption der Idee und die Wirklichkeit, edited by Zenon H. Nowak and Roman Czaja, 39-53. Torun 2001.

Arnold, Udo. Deutscher Orden und Preußenland. Ausgewäblte Aufsätze anläßlich des 65. Geburtstages, edited by Bernhart Jähnig and Georg Michels. Marburg: Elwert, 2005.

Arnold, Udo. "Dr. Marian Tumler." In Die Hochmeister des Deutschen Ordens IIgo-20I2, edited by Udo Arnold, 330-336. Weimar: VDG, 2014.

Arnold, Udo. "Hochmeister Albrecht von Brandenburg-Ansbach und Landmeister Gotthard Kettler - Ordensritter und Territorialherren am Scheideweg in Preußen und 
Livland." In The Military Orders and the Reformation. Choices, State building, and the Weight of Tradition. Papers of the Utrecht Conference, edited by Johannes A. Mol, Klaus Militzer and Hellen Nicholson, I I -29. Hilversum: Uitgeverij Verloren, 2006.

Arnold, Udo. "Hochmeister P. Dr. Marian Tumler ( I 887- I 987)." In Priester im Deutschen Orden.Vorträge der Tagung der Internationalen Historischen Kommission zur Erforschung des Deutschen Ordens in Wien 2012, edited by Udo Arnold, 21 5-230. Weimar: VDG, 2016.

Arnold, Udo. "Hochmeisterverlust, Bauernunruhen und Reformation - Krisenbewältigung unter den Deutschmeistern Dietrich von Cleen und Walter von Cronberg." In Die Ritterorden in Umbruchs- und Krisenzeiten. The Military Orders in Times of Change and Crisis, edited by Roman Czaja und Jürgen Sarnowsky, 24 I-257. Toruń: Wydawnictwo Naukowe UMK, 20 I I.

Arnold, Udo. "Nationalismus, Nationalsozialismus und der Mißbrauch der Deutschordenstradition in Deutschland." In Der Deutsche Orden und die Ballei Elsaß-Burgund. Die Freiburger Vorträge zur 80o-Jahr-Feier des Deutschen Ordens, edited by Hermann Brommer, 205-222. Bühl/Baden: Konkordia Verlag, I 996.

Arnold, Udo., Huber, Bernhard., edit. Peregrinantes Peregrinantibis. 825 Jahre Deutscher Orden, Iso Jahre Ehrenritter, so Jahre Familiarenstatut. Weimar: VDG, 20 I 8.

Arnold, Udo. Reformatorskie tendencje w zakonie krzyżackim w Prusach w I potowie XV wieku. Zapiski Historyczne 45 (1980), 4: 7-19.

Arnold, Udo. "Reformansätze im Deutschen Orden während des Spätmittelalters." In Reformbemühungen und Observanzbestrebungen im spätmittelalterlichen Ordenswesen, edited by Kaspar Elm, I39-I 52. Berlin: Duncker \& Humblot, I 989.

Bruin, Renger E. de. Bedreigd door Napoleon. De Ridderlijke Duitsche Orde, Balije van Utrecht I753-I838. Hilversum: Uitgeverij Verloren, $20 \mathrm{I} 2$.

Demel, Bernhard. "Die bi- und schließlich trikonfessionelle Ballei Hessen des Deutschen Ordens zwischen Luther und Napoleon und die Ordenspfarre Reichenbach in Nordhessen von I 207 bis I 809 ." In II90-2010. 820 Jahre Deutscher Orden. Fünf Referate über eine lange Geschichte in Europa, edited by Bernhard Demel. 26-79. Wien: Riedeldruck, $201 \mathrm{I}$.

Demel, Bernhard. "Der Deutsche Orden und das Regiment Hoch- und Deutschmeister von I 696 bis 1 998 . Überblick und neue Erkenntnisse." In Der Deutsche Orden einst und jetzt. Aufsätze zu seiner mehr als 8oojährigen Geschichte, edited by Bernhard Demel and Friedrich Vogel, 85 - i i s. Frankfurt/Main: Peter Lang, I 999.

Demel, Benhard. Das Priesterseminar des Deutschen Ordens zu Mergentheim. Bonn-Bad Godesberg: Wissenschaftliches Archiv, I 972.

Demel, Bernhard. "Rekuperationsbemühungen des Deutschen Ordens um die ihm entzogene Ballei Utrecht in den Jahren I 667-1 68 5.” In Flores considerationum amicorum. Festschrift für Carl August Lückerath zum 70. Geburtstag am I3. Dezember 2006, edited by Wolfgang Hasberg und Josef Schröder, 8 I - I 03. Zürich: Muster-Schmidt, 2006.

Demel, Bernhard. "Von der katholischen zur trikonfessionellen Ordensprovinz. Entwicklungslinien in der Personalstruktur der hessischen Deutschordensballei in den Jahren 
I 526-1680/8 I." In Elisabeth, der Deutsche Orden und ihre Kirche. Festschrift zur 7oojährigen Wiederkehr der Weihe der Elisabethkirche Marburg I983, edited by Udo Arnold and Heinz Liebing, I 86-28 I. Marburg: Elwert, 1983.

Demel, Bernhard. "Zur Priestergewinnung und Seminarausbildung im Deutschen Reich und im schlesisch-mährischen Meistertum zwischen Reformation und Säkularisation.” In Priester im Deutschen Orden. Vorträge der Tagung der Internationalen Historischen Kommission zur Erforschung des Deutschen Ordens in Wien 2012, edited by Udo Arnold, I 57-I 75. Weimar: VDG, 2016.

Gasser, Urlich. Die Priesterkonvente des Deutschen Ordens. Peter Rigler und ibre Wiedererrichtung I854-1897. Bonn-Bad Godesberg: Wissenschaftliches Archiv, 1973.

Grögor-Schiemann, Daniela. Die Deutschordensballei Utrecht während der Reformationszeit: Die Landkommende zwischen Rebellion und Staatsbildung. Weimar: VDG, 2015.

Gruber, Erentraud. Deutschordensschwestern im 19. und 20. Jabrhundert. Wiederbelebung, Ausbreitung und Tätigkeit I837-1971. Bonn-Bad Godesberg: Wissenschaftliches Archiv, 197 I.

Gruber, Erentraud. Hinter dem Eisernen Vorhang. Deutschordensschwestern in der ČSR/ČSSR 1945 bis 1989 . Weimar: VDG, $20 \mathrm{I} 2$.

Herrmann, Axel. Der Deutsche Orden unter Walter von Cronberg (I525-I543). Zur Politik und Struktur des „Teutschen Adels Spitale“ im Reformationszeitalter. Bonn-Bad Godesberg: Wissenschaftliches Archiv, 1974.

Militzer, Klaus. Die Geschichte des Deutschen Ordens. Stuttgart: Kohlhammer, ${ }^{2} 20$ I 2.

Müller, Gerard. Die Familiaren des Deutschen Ordens. Marburg: Elwert, ${ }^{2} 20$ I o.

Noflatscher, Heinz. Glaube, Reich und Dynastie. Maximilian der Deutschmeister 1558$-{ }_{-16} 8$. Marburg: Elwert, 987.

Platter, Bruno. "Werdegang und Charakterisierung der nachkonziliaren Schwesternregeln." In Die Regeln des Deutschen Ordens in Geschichte und Gegewart, edited by Ewalt Volgger, 303-327. Lana: Deutschordens-Verlag, 1985.

Täubl, Friedrich. Der Deutsche Orden im Zeitalter Napoleons. Bonn: Wissenschaftliches Archiv, 1966.

Volgger, Ewald., edit. Erzherzog Maximilian Joseph von Österreich-Este. Hochmeister - Festungsplaner - Sozialreformer - Bildungsforderer (1782-1863). Mit Beiträgen zum Deutschen Orden in Linz und Oberösterreich. Linz-Weimar: VDG, 20 I 4.

Wieland, Arnold O. "Wandel im 20. Jahrhundert: Vom Ritterorden zum klerikalen Orden." In Priester im Deutschen Orden. Vorträge der Tagung der Internationalen Historischen Kommission zur Erforschung des Deutschen Ordens in Wien 2012, edited by Udo Arnold, I 77-2 I 4. Weimar: VDG, 2016. 


\section{ABSTRACT \\ Reform and Renewal in the Teutonic Order $16^{\text {th }}-20^{\text {th }}$ century}

The turning-points in the 16th century made renewals necessary. After loosing Prussia in 1525 the German Master took over the leadership, not the Master of Livonia. He united the bailiwicks in the German Empire, but Livonia was an appendix until 1561/1562, when it was lost too. The German Master was a Prince of the Empire, and the Order had the new mission of fighting against the Turks, as a support of the Empire.

The German reformation divided the principalities into Catholics and Lutherans. The Order had possessions in both, so the brethren became also members of the different confessions. After 1648, when the confession of Calvin became legal, the Order was tridenominational, for instance in the bailiwick of Hessia. The bailiwick of Utrecht separated and became an independant branche of the Order, until today in the Netherlands.

In the 17 th century the Order got a new rule, which gave more freedom to the knights. In the same time the Master reformed the education of the priests by erecting an own seminar in Mergentheim, similar in other regions.

After the expulsion from the German Empire by Napoleon the Order existed only in the Habsburg Empire with an archiduke as Master and as an Order of the Roman church. He got a new rule and the renewal of the knights in 1839, the sisters and the priests in 1855. He erected branches of laity, named Knights of Honor and Marianer; they helped to finance the hospitals of the Order, especially in the wars.

After the breakdown of the Habsburg Empire the Order has been renewed by the Roman church as a catholic Order of priests, brethren, sisters and laity, without knights, in 1929. Today he is working as an clerical and a social welfaring Order in seven European states. 